(2) Open Access Full Text Article

\title{
Irinotecan-Induced Skin Dryness Is Ameliorated By Orally Administered High-Dose Vitamin C In Mice
}

\author{
Kentaro Nakanishi ${ }^{1}$ \\ Kenji Goto (D) \\ Kanako Kondo ${ }^{1,2}$ \\ Keiichi Hiramoto' \\ Kazuya Ooi \\ 'Department of Pharmaceutical Science, \\ Suzuka University of Medical Science, \\ Suzuka, Mie 5I3-8670, Japan; \\ ${ }^{2}$ Department of Pharmacy, Kuwana City \\ Medical Center, Kuwana, Mie 5II-006I, \\ Japan
}

This article was published in the following Dove Press journal: Journal of Experimental Pharmacology

Background: Vitamin C plays a part in various roles in the human body. In this study, we examined the effect of oral administration of high-dose vitamin $\mathrm{C}$ on the skin dryness induced by irinotecan.

Methods: To establish the experimental model of irinotecan-induced skin dryness, the drug was intraperitoneally administered for four consecutive days. Simultaneously, oral administration of high-dose vitamin C (4 g/ $\mathrm{kg})$ was continued for 4 days.

Results: High-dose vitamin $\mathrm{C}$ administration ameliorated the skin dryness induced by irinotecan. The expression of caspase- 3 and caspase-9, reactive oxygen species, and the number of TUNEL-positive cells increased in the skin of irinotecan-treated mice but were lowered by high-dose vitamin $\mathrm{C}$ administration. In contrast, fibroblasts and collagen type I decreased in the skin of the irinotecan-treated mice but was increased by high-dose vitamin $\mathrm{C}$ administration.

Conclusion: These results suggested that high-dose vitamin $\mathrm{C}$ administration can improve the skin dryness induced by irinotecan.

Keywords: apoptosis, collagen type I, irinotecan, reactive oxygen species, vitamin C

\section{Introduction}

A cancer cell reports a disorderly proliferation. DNA replication is necessary for cell proliferation. Irinotecan is activated upon hydrolyzation by $\mathrm{SN}-38$ and obstructs topoisomerase $\mathrm{I}$, which is necessary for DNA replication. ${ }^{1,2}$ The drug acts not only on cancer cells but also on healthy cells, thus killing them. Furthermore, it is known to have various side effects, such as leukopenia, ${ }^{3,4}$ loose intestines, ${ }^{5}$ and the psilosis. ${ }^{6}$ Irinotecan also causes skin dryness ${ }^{3}$ have become a course of the infective disease and the allergy. ${ }^{7}$ However, skin dryness induced by irinotecan has not been studied in detail.

Vitamin $\mathrm{C}$ is well known for its antioxidant action. ${ }^{8}$ Furthermore, a vitamin $\mathrm{C}$ has an effect in prophylaxis of scorbutus with a compound necessary for generation of the collagen which is connective protein. ${ }^{9}$ In addition, high-dose vitamin $\mathrm{C}$ administration has been reported to have anticancer effects. ${ }^{10,11}$ We also reported that high-dose vitamin $\mathrm{C}$ administration has an ameliorative effect against colon cancer induced by azoxymethane and dextran sodium sulfate. ${ }^{12}$ In this study, we examined the mechanism by which irinotecan induces skin dryness, and whether high-dose vitamin $\mathrm{C}$ administration has an ameliorative effect against skin dryness.
Correspondence: Kazuya Ooi Department of Pharmaceutical Science, Suzuka University of Medical Science, Suzuka, Mie 513-8670, Japan

Email zooi@suzuka-u.ac.jp 


\section{Materials And Methods}

\section{Experimental Animals}

Male hairless mice (HR-1; 7-week-old) were obtained from SLC (Hamamatsu, Shizuoka, Japan). The animals were housed under a 12-h light/dark cycle, a constant temperature of $23 \pm 1{ }^{\circ} \mathrm{C}$, and $50 \pm 10 \%$ relative humidity. The mice were allowed free access to laboratory chow and water. After an acclimation period of 1 week, the mice were randomly assigned to four groups ( $\mathrm{n}=5$ /group). Group 1 was the control group; Group 2 included animals that were administered high-dose vitamin C (Fuso Pharmaceutical Industries Ltd., Osaka, Japan); Group 3 included animals that were administered irinotecan (Daiichi Sankyo Healthcare Co., Ltd., Tokyo, Japan); and Group 4 comprised animals that were administered irinotecan and vitamin C. We performed repeated administration of vitamin C $(100 \mathrm{mg} / \mathrm{mouse} /$ day $)$ and irinotecan $(60 \mathrm{mg} / \mathrm{kg} /$ day $)$ once per day for 4 days. These reagents were diluted with distilled water. Furthermore, vitamin $\mathrm{C}$ was administered through the oral route and irinotecan through the intraperitoneal route. ${ }^{12}$ Each of these solutions was administered at $0.2 \mathrm{~mL}$. On the fifth day of the study, dorsal skin samples were obtained. The study was carried out in strict accordance with the recommendations of the Guide for the Care and Use of Laboratory Animals of Suzuka University of Medical Science (approval number: 34). All surgeries were performed under pentobarbital anesthesia and every effort was made to minimize animal suffering.

\section{Measurement Of Transepidermal Water Loss}

Transepidermal water loss (TEWL) from the dorsal skin was measured. TEWL measurements were obtained to determine skin permeability (reflecting the barrier function of the skin) by using Tewameter TM300 (Courage+Khazaka Electronic $\mathrm{GmbH}$, Cologne, Germany), as described previously. ${ }^{13}$ TEWL serves as a marker of skin permeability, reflecting the barrier function of the skin; increased TEWL indicates dry skin.

\section{Skin Staining}

The fixed skin specimens were cut into 5 - $\mu$ m-thick sections. The sections were then stained with hematoxylin and eosin (H\&E) for histopathological analysis of the tissue in accordance with established procedures. The other specimens were used to analyze apoptosis. Apoptosis was evaluated in situ in colon sections with an apoptosis detection kit
(Takara Biomedicals, Tokyo, Japan). The number of terminal deoxynucleotidyl transferase dUTP nick end labeling (TUNEL)-positive cells was determined using the imageprocessing software Image $\mathrm{J}$ (National Institutes of Health, Bethesda, MD, USA).

\section{Western Blot Detection Of Collagen Type I, Caspase-3, Caspase-9, And Fibroblasts In The Skin}

Western blotting was performed as previously described. ${ }^{14}$ Briefly, the samples were separated by electrophoresis and the membranes were incubated at $25^{\circ} \mathrm{C}$ for $1 \mathrm{~h}$ with primary antibodies against S100A4, a fibroblast-specific marker (1:1000; RB-1804-A0, Thermo Fisher Scientific, Waltham, MA, USA), collagen type I (1:1000; 234,167, Millipore, Billerica, MA, USA), cleaved caspase-3 (1:1000; Cell Signaling Technology, Danvers, MA, USA), cleaved caspase-9 (1:1000; Cell Signaling Technology), or $\beta$-actin as the loading control (1:5000; A5441-100UL, Sigma-Aldrich Corp., St. Louis, MO, USA). The membranes were then treated with a horseradish peroxidase-conjugated secondary antibody (1:1000; Novex, Frederick, MD, USA). Images of the membranes were acquired with the Multi-Gauge Software Program (Fujifilm, Greenwood, SC, USA).

\section{Quantification Of Skin ROS Level}

The skin reactive oxygen species (ROS) level was determined with an OxiSelect ${ }^{\mathrm{TM}}$ STA-347 in vivo ROS/RNS assay kit (Cell Biolabs, Inc., San Diego, CA, USA) in accordance with the manufacturer's instructions.

\section{Statistical Analyses}

All data are presented as mean \pm standard deviation (SD) value. The results were analyzed with Microsoft Excel 2010 software (Microsoft Corp., Redmond, WA, USA). Differences between the groups were evaluated by one-way analysis of variance followed by Tukey's post-hoc parametric test using SPSS version 20 software (SPSS, Inc., Chicago, IL, USA). The results were considered significant at $p<0.05$.

\section{Results}

\section{Effects Of High-Dose Vitamin C} Administration On Change In Skin Status In The Mice

We measured TEWL from the dorsal skin to evaluate skin dryness. TEWL significantly increased in the 
irinotecan administration group; the remaining three groups did not show a change (Figure 1A). Further, we examined histological changes in the skin by $\mathrm{H} \& \mathrm{E}$ staining. Skin thickness decreased in the irinotecan administration group. Skin thickness in the vitamin C plus irinotecan administration group was not different from that in the control and vitamin $\mathrm{C}$ administration groups (Figure 1B). In addition, the expression of skin collagen type $\mathrm{I}$, which is a supporting tissue in the skin, was significantly lowered by the irinotecan administration. The expression of collagen type $\mathrm{I}$ in the irinotecan plus vitamin $\mathrm{C}$ administration group was not different from that in the control and vitamin $\mathrm{C}$ administration groups (Figure 1C).
Effects Of High-Dose Vitamin C Administration On The Expression Of Caspase-3, Caspase-9, Fibroblast, And Apoptosis In The Skin Of The Mice

We examined caspases associated with apoptosis and fibroblasts. Caspases include pro-caspase and activated (cleaved) caspase. The pro-caspase forms are always present in cells, and their levels do not relate to apoptosis. Therefore, we confirmed the expression of cleaved caspases 3 and 9 in this study. Caspase-3 and caspase- 9 levels increased most by irinotecan administration: levels in the irinotecan plus vitamin $\mathrm{C}$ administration group were significantly lower than those in the irinotecan administration group but were higher than those in the control and vitamin
A

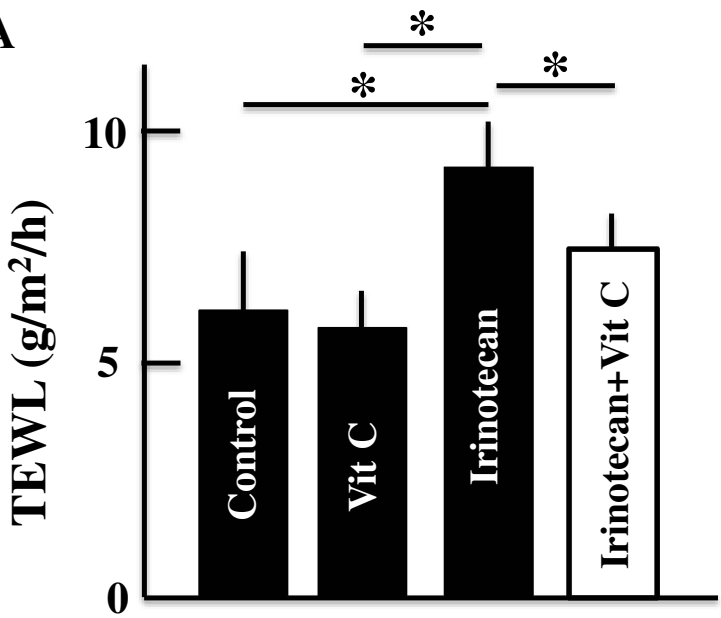

C
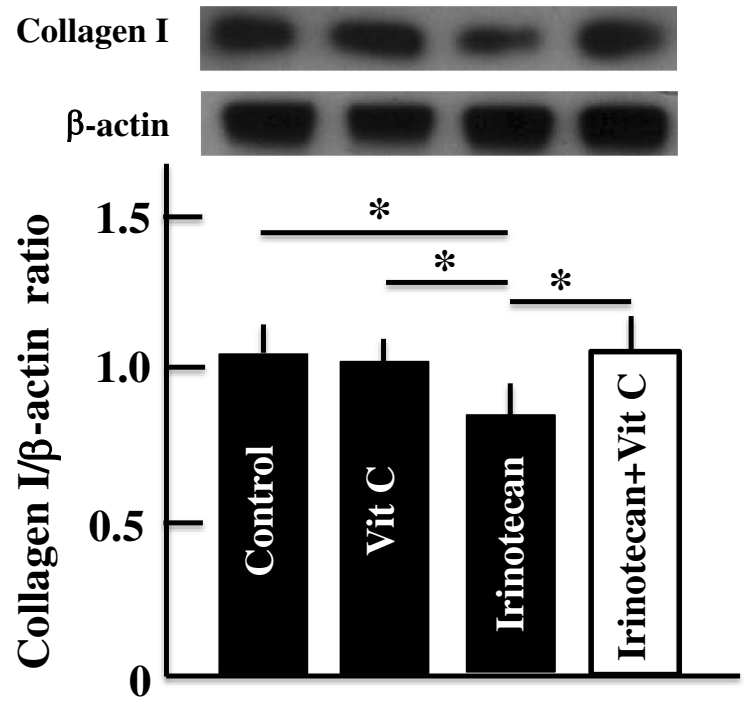

B

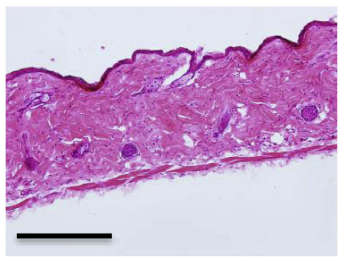

Control

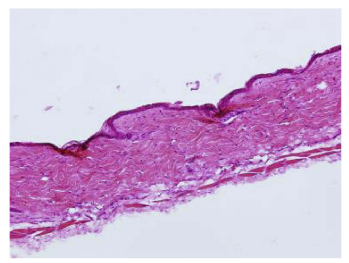

Irinotecan

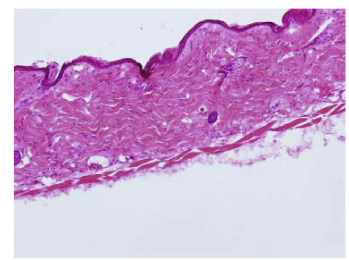

Vit C

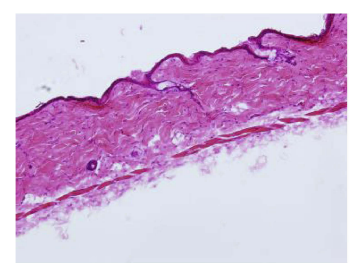

Irinotecan+Vit C

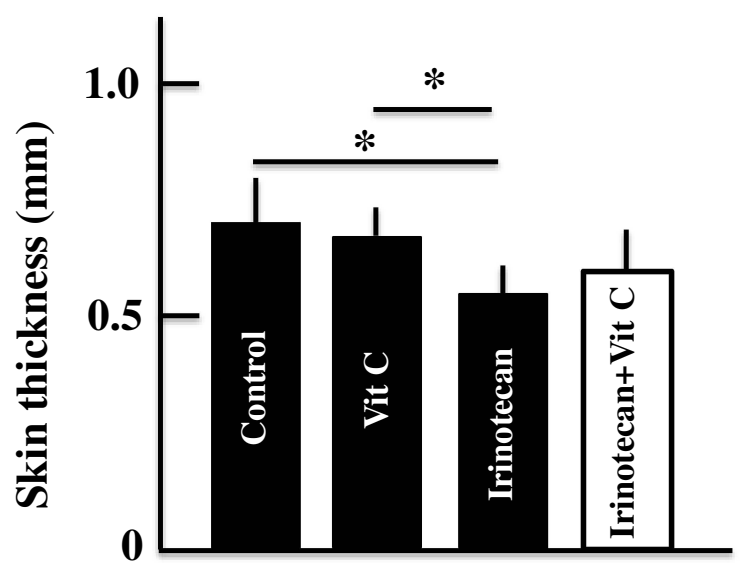

Figure I Effects of high-dose vitamin C administration on irinotecan-induced skin dryness. TEWL (A), skin thickness (B), and the expression of collagen type I in the skin (C). Values are presented as the mean \pm SD values of six animals. $* P<0.05$. Scale bar $=100 \mu \mathrm{m}$.

Abbreviation: Vit C, vitamin C. 
$\mathrm{C}$ administration groups (Figure 2A and $\mathrm{B}$ ). On the other hand, the number of fibroblasts in the skin decreased in the irinotecan administration group but increased in the irinotecan plus vitamin $\mathrm{C}$ administration group; however, they were not different those in the control group (Figure 2C). The number of TUNEL-positive cells significantly increased after irinotecan administration. However, the number was not different between the control, Vitamin $\mathrm{C}$, and irinotecan plus vitamin $\mathrm{C}$ administration groups (Figure 2D).

\section{Effects Of High-Dose Vitamin C Administration On ROS Levels In Mouse Skin}

ROS levels in mouse skin significantly increased after irinotecan administration. The ROS levels in the irinotecan plus vitamin $\mathrm{C}$ administration group were lower than those in the irinotecan administration group but were higher than those in the control and vitamin $\mathrm{C}$ administration groups (Figure 3).

\section{Discussion}

In this study, an increase in TEWL, a decrease in skin thickness, and a decrease in the expression of collagen type I were observed after administration of irinotecan, and skin dryness was induced. These levels approached those in the control group after the administration of high-dose vitamin $\mathrm{C}$, and amelioration of skin dryness was noted. Furthermore, after the administration of irinotecan, an increase in ROS, caspase-3, and caspase-9 levels; a decrease in the number of fibroblasts; and induction of apoptosis were observed. On the other hand, vitamin $\mathrm{C}$ administration ameliorated these parameters.

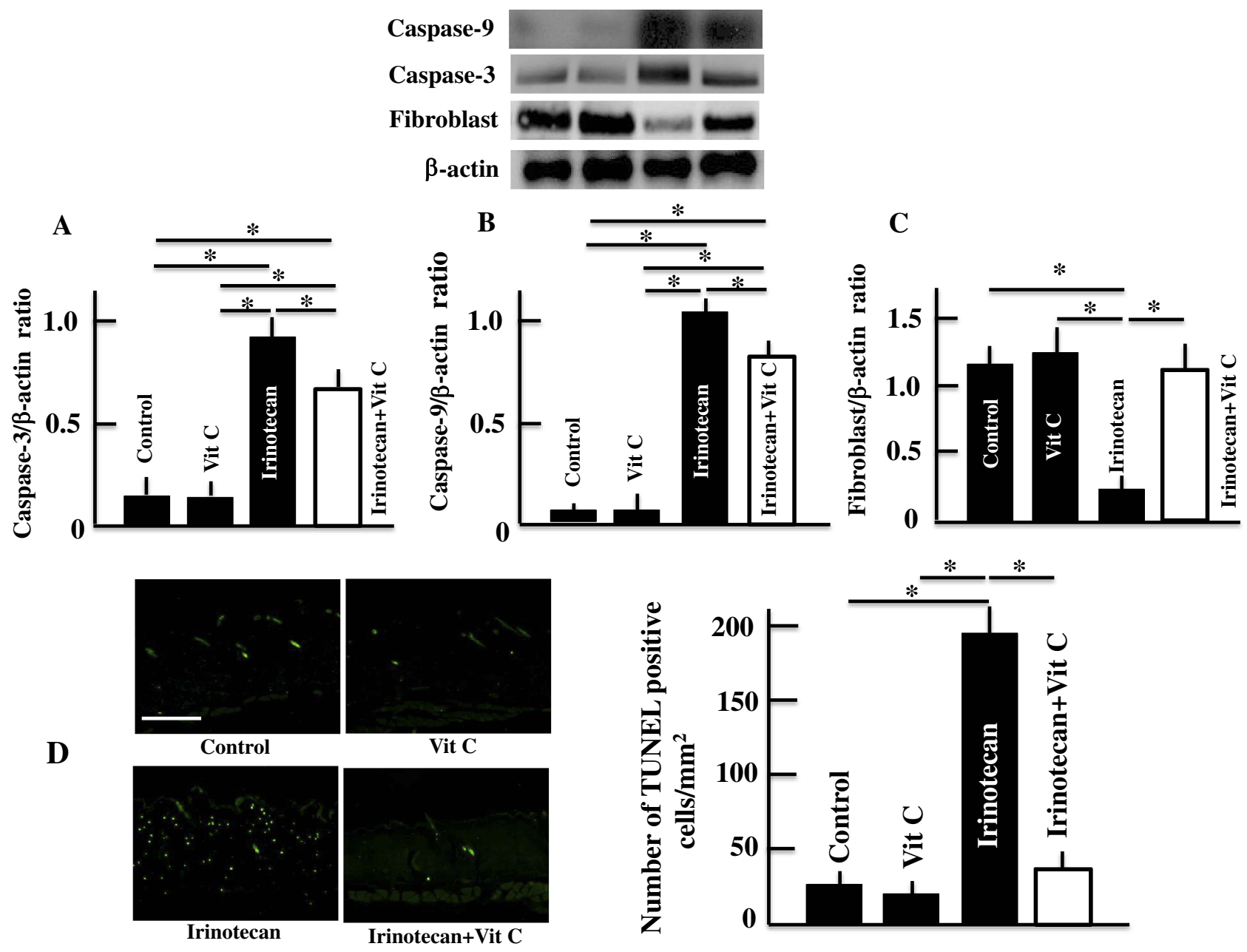

Figure 2 Effects of high-dose vitamin C administration on caspase-3 (A), caspase-9 (B), fibroblasts (C), and TUNEL-positive cells (D) in the skin. Values are presented as the mean \pm SD values of six animals. $* P<0.05$. Scale bar $=100 \mu \mathrm{m}$.

Abbreviation: Vit $C$, vitamin $C$. 


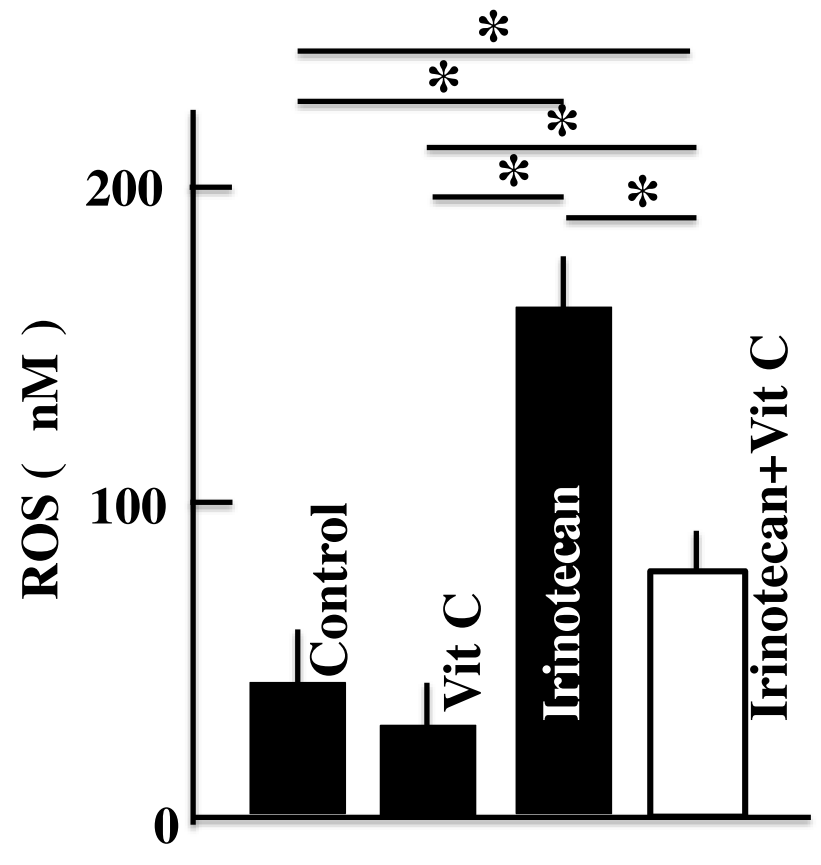

Figure 3 Effects of high-dose vitamin $C$ administration on skin levels of ROS in the mice. Values are presented as the mean \pm SD values of six animals. $* P<0.05$. Abbreviation: Vit C, vitamin C.

The administration of an anticancer agent affects not only cancer cells but also healthy cells, results in the induction of apoptosis and eventually destruction a cell. ${ }^{15-18}$ Stimulation by anticancer agents activates BCL2 associated X (Bax). Bax is taken into the mitochondrial membrane and leads to the release the cytochrome $\mathrm{C}$. The released cytochrome $\mathrm{C}$ combines with apoptotic protease-activating factor (Apaf)-1 and activates caspase-9. Finally, caspase-9 activates caspase-3, which causes DNA fragmentation and induces apoptosis. ${ }^{15-18}$ The activation of caspase-9 and caspase-3 occurred thusly after irinotecan administration in this study, and was followed by the induction of apoptosis (Figure 2A, B, and D). Furthermore, the number of fibroblasts decrease (Figure 2C). Based on these findings, it was thought that the apoptosis of the fibroblasts, which generate collagen type I, was induced and collagen type I expression decrease. In addition, it has been reported that ROS generation occurs in the induction process of apoptosis mediated by an anticancer agent. ${ }^{19-23}$

Vitamin $\mathrm{C}$ exhibits an antioxidant effect by reducing free radicals and oxygen-derived species such as hydroxyl radicals and hydrogen peroxide. ${ }^{24}$ The abovementioned findings suggested that regard to the amelioration of skin dryness by high-dose vitamin $\mathrm{C}$ administration, vitamin $\mathrm{C}$ administration inhibited apoptosis induction in fibroblasts by inhibiting ROS generation. ROS directly stimulate fibroblasts. Stimulation of fibroblasts promotes the production of matrix metalloproteinase-1 and decomposition of collagen. ${ }^{25}$ High-dose vitamin $\mathrm{C}$ may suppress the degradation of collagen type I by inhibiting ROS production. Furthermore, it was thought to facilitate damage recovery in the skin and ameliorate skin dryness by ensuring the production of collagen type $\mathrm{I}^{26}$ However, further studies are warranted because vitamin $\mathrm{C}$ has not only antioxidant function but also cofactor activity.

\section{Conclusions}

Skin dryness is a side effect of irinotecan. It was caused by damage to the mitochondria and ROS generation, which was caused by cell destruction due to apoptosis induced by irinotecan. Oral administration of high-dose vitamin $\mathrm{C}$ inhibited the induction of the apoptosis and ROS generation mediated by irinotecan. Thus, it can be used to care for the skin of patients receiving anticancer drug treatment and can improve patients' quality of life. However, since this study has been performed in mice, clinical testing in humans is necessary.

\section{Abbreviations}

TEWL, transepidermal water loss; TUNEL, transferase dUTP nick end labeling; ROS, reactive oxygen species; Bax, BCL2 associated X; Apaf, apoptotic protease-activating factor.

\section{Acknowledgment}

This study was supported by Grant-in-Aid for Scientific Research (C) from the Japan Society for the Promotion of Science (JSPS; Grant number: 18K06802).

\section{Disclosure}

The authors report no conflicts of interest in this work.

\section{References}

1. Nitta K, Yokokura T, Sawada S, et al. Antitumor activity at new derivatives of camptothecin. Gan To Kagaku Rtoho. 1987;14:850-857.

2. Kawato Y, Aonuma M, Hirota Y, Kuga H, Sato K. Intracellular roles of $\mathrm{SN}-38$, a metabolite of the camptothecin derivative CPT-11, in the antitumor effect of CPT-11. Cancer Res. 1991;51:4187-4191.

3. Tanaka Y, Katoh M, Fujioka M, et al. Effect of tacrolimus on the pharmaceutics and glucuronidation of SN-18, an active metabolite of irinotecan. Yakugaku Zasshi. 2013;133:463-471. doi:10.1248/yakushi.12-00276

4. Yokoyama S, Kouyama M, Kuratsune M, et al. A case of an advanced gastric cancer patient on hemodialysis achieving long-term progression-free survival after CPT-11+CDDP therapy. Gan To Kagaku Ryoho. 2012;39:817-820.

5. Araki E, Ishikaea M, Ligo M, Itabashi M, Koshi A. Relationship between development of diarrhea and the concentration of SM-38, an active metabolite of CPT-11, in the intestine and the blood plasma of athymic mice following intraperitoneal administration of CPT-11. Jpn J Cancer Res. 1993;84:697-702. doi:10.1111/j.1349-7006.1993.tb02031.x 
6. Arai Y, Ohtsu A, Sato Y, et al. Phase I/II study of radiologic hepatic arterial infusion of fluorouracil plus systemic irinotecan for unresectable hepatic metastases from colorectal cancer: Japan Clinical Oncology Group Trial 0208-DI. J Vasc Interv Radiol. 2012;23:1261-1267. doi:10.1016/j.jvir.2012.06.031

7. Paller AS, Simpson EL, Eichenfield LF, Ellis CN, Mancini AJ. Treatment strategies for atopic dermatitis: optimizing the available therapeutic options. Semin Cutan Med Surg. 2012;31:S10-S17. doi:10.1016/j.sder.2012.07.004

8. Frei B, England L, Ames BN. Ascorbate is an outstanding antioxidant in human blood plasma. Proc Natl Acad Sci USA. 1989;86:6377-6381. doi:10.1073/pnas.86.16.6377

9. Li Y, Schellhorn HE. New developments and novel therapeutic perspectives for vitamin C. J Nutr. 2007;137:2171-2184. doi:10.1093/jn/ 137.10.2171

10. Padayatty SJ, Riordan HD, Hewitt SM, Katz A, Hoffer LJ, Levine M. Intravenously administered vitamin $\mathrm{C}$ as cancer therapy: three cases. CMAJ. 2006;174:937-942. doi:10.1503/cmaj.050346

11. Levine M, Espey MG, Chen Q. Losing and finding a way at C: new promise for pharmacologic ascorbate in cancer treatment. Free Radic Biol Med. 2009;47:27-29. doi:10.1016/j.freeradbiomed.2009.04.001

12. Kondo K, Sano R, Goto K, Hiramoto K, Ooi K. Administration of high-dose vitamin $\mathrm{C}$ and irinotecan ameliorates colorectal cancer induced by azoxymethane and dextran sodium sulfate in mice. Biol Pharm Bull. 2018;41:1797-1803. doi:10.1248/bpb.b18-00453

13. Barel AD, Clarys P. Study of the stratum corneum barrier function by transepidermal water loss measurement: comparison between two commercial instruments: evaporimeter and tewameter. Skin Pharmacol. 1995;8:186-195.

14. Yokoyama S, Hiramoto K, Koyama M, Ooi K. Chronic liver injury in mice promotes impairment of skin barrier function via tumor necrosis factor-alpha. Cutan Ocul Toxicol. 2016;35:194-203. doi:10.3109/ 15569527.2015 .1076433

15. Stennicke HR, Salvesen GS. Properties of the caspases. Biochim Biophys Acta. 1998;1387:17-31. doi:10.1016/s0167-4838(98)00133-2

16. Thornberry NA, Lazebnik Y. Caspases: enemies within. Science. 1998;281:1312-1316. doi:10.1126/science.281.5381.1312
17. Earnshaw WC, Martins LM, Kaufmann SH. Mammalian caspase: structure, activation, substrates, and functions during apoptosis. Annu Rev Biochem. 1999;68:383-424. doi:10.1146/annurev.biochem.68.1.383

18. Sakamaki K, Satou Y. Caspase: evolutionary aspects of their functions in vertebrates. J Fish Biol. 2009;74:727-753. doi:10.1111/ j.1095-8649.2009.02184.x

19. Gewirtz DA. A critical evaluation of the mechanisms of action proposed for the antitumor effects of the anthracycline antibiotics adriamycin and daunorubicin. Biochem Pharmacol. 1999;57:727741. doi:10.1016/s0006-2952(98)00307-4

20. Hiraoka W, Vazquez N, Nieves-Neira W, Chanock SJ, Pommier Y. Role of oxygen radicals generated by NADPH oxidase in apoptosis induced in human leukemia cells. J Clin Invest. 1998;102:19611968. doi:10.1172/JCI3437

21. Tada-Oikawa S, Oikawa S, Kawanishi M, Yamada M, Kawanishi A. Generation of hydrogen peroxide precedes loss of mitochondrial membrane potential during DNA alkylation-induced apoptosis. FEBS Lett. 1999;442:65-69. doi:10.1016/s0014-5793(98) 01618-4

22. Varbiro G, Veres B, Gallyas F Jr, Sumegi B. Direct effect of Taxol on free radical formation and mitochondrial permeability transition. Free Radic Biol Med. 2001;31:548-558. doi:10.1016/s0891-5849(01) 00616-5

23. Murata M, Suzuki T, Midorikawa K, Oikawa S, Kawanishi S. Oxidative DNA damage induced by a hydroperoxide derivative of cyclophosphamide. Free Radic Biol Med. 2004;37:793-802. doi:10.1016/j. freeradbiomed.2004.05.009

24. Arrigoni O, De Tullio MC. Ascorbic acid: much more than just an antioxidant. Biochim Biophys Acta. 2002;1569:1-9. doi:10.1016/ s0304-4165(01)00235-5

25. Hwang E, Park SY, Lee HJ, Lee TY, Sun ZW, Yi TH. Gallic acid regulates skin photoaging in UVB-exposed fibroblast and hairless mice. Phytother Res. 2014;28:1778-1788. doi:10.1002/ptr.5198

26. Ivanov SV, Ivanov IS, Tsukanov AV, Gafarov GN, Obyedkov EG. The influence of vitamin $\mathrm{C}$ on the dynamic of relation of collagen I and III types in the periprosthesis area. Tsitologiia. 2016;58:714-719.

\section{Publish your work in this journal}

The Journal of Experimental Pharmacology is an international, peerreviewed, open access journal publishing original research, reports, reviews and commentaries on all areas of laboratory and experimental pharmacology. The manuscript management system is completely online and includes a very quick and fair peer-review system. Visit http://www.dovepress.com/testimonials.php to read real quotes from published authors. 